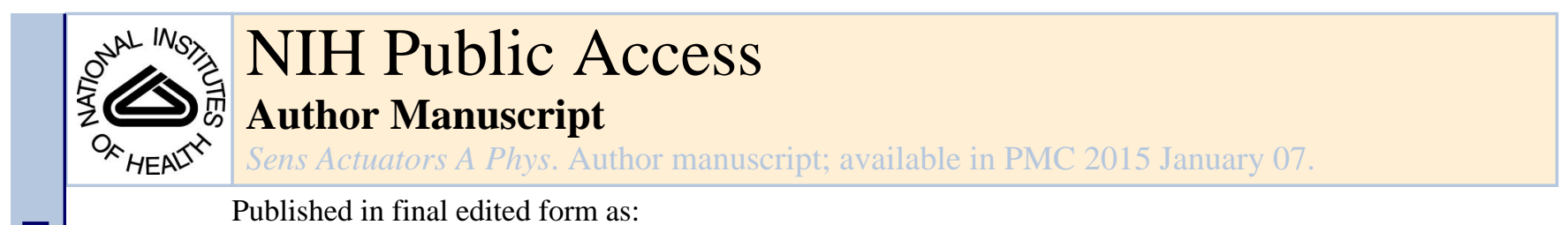

Published in final edited form as:

Sens Actuators A Phys. 2009 March 16; 150(1): 144-150. doi:10.1016/j.sna.2008.11.008.

\title{
Micromachined "Side-Viewing" Optical Sensor Probe for Detection of Esophageal Cancers
}

\author{
A. Garcia-Uribe ${ }^{1,{ }^{*}}$, K. C. Balareddy ${ }^{1,},{ }^{*}$ J. Zou ${ }^{1}$, A. K. Wojcik ${ }^{1}$, K. K. Wang ${ }^{2}$, and L. V. Wang ${ }^{3}$ \\ ${ }^{1}$ Department of Electrical and Computer Engineering, Texas A\&M University, College Station, TX \\ 77843, USA \\ ${ }^{2}$ Mayo Clinic, Rochester, Minnesota 55905, USA \\ ${ }^{3}$ Department of Biomedical Engineering, Washington University in St. Louis, MO 63130, USA
}

\begin{abstract}
In this paper, we report the design, fabrication and testing of a new miniaturized optical sensor probe with "side viewing" capability for oblique incidence diffuse reflectance spectrometry. The sensor probe consists of a lithographically patterned polymer waveguides chip and two micromachined positioning substrates and source/collection fibers to achieve $45^{\circ}$ light incidence and collection of spatially resolved diffuse reflectance. Diffuse reflectance of human esophageal surface has been successfully measured for differentiation of cancerous tissues from normal ones.
\end{abstract}

\section{Keywords}

Micromachined probe; oblique incidence; diffuse reflectance; esophageal cancer

\section{INTRODUCTION}

Esophageal cancers are in most cases lethal malignant lesions of the human esophagus. According to the American Cancer Society, there were around 16,470 new cases of esophageal cancers with about 14,280 deaths in 2008 [1]. Some countries such as Iran, northern China, India, and southern Africa have occurrence rates 10-100 times higher than that of the United States [1]. Because esophageal cancer is usually diagnosed at a late stage, most people with esophageal cancer eventually die of this disease. Therefore, there is an urgent need for early diagnosis techniques, which are accurate, non-invasive and low-cost. Recent study has shown that the optical properties (absorption and scattering) of human tissue are closely related to some of the key diagnostic physiological parameters, which would significantly vary with its exact disease state [2-8].

\footnotetext{
*These two authors equally contribute to this work.

Publisher's Disclaimer: This is a PDF file of an unedited manuscript that has been accepted for publication. As a service to our customers we are providing this early version of the manuscript. The manuscript will undergo copyediting, typesetting, and review of the resulting proof before it is published in its final form. Please note that during the production process errors may be discovered which could affect the content, and all legal disclaimers that apply to the journal pertain.
} 
Oblique incidence diffuse reflectance spectroscopy (OIDRS) is a non-invasive optical method, which utilizes a special fiber optic sensor probe to measure the diffuse reflectance of inhomogeneous media (e.g. biological tissues), from which the absorption and reduced scattering coefficients can be readily extracted [9]. Rapid and accurate (automated) classification of precancerous and invasive cancerous skin abnormalities could be achieved based on real-time processing of OIDRS images [10]. This result indicates that OIDRS could be a premium noninvasive imaging method for accurate detection of human cancers, especially those occurring on the surfaces of tissues or organs. Unfortunately, OIDRS is currently limited to only skin applications by the available sensor probes, which have only "front viewing" capability (Fig. 1a) [11]. To significantly extend OIDRS for the detection of a much wider variety of cancers, new sensor probes with smaller size and more importantly "side viewing" capability are necessary for effective OIDRS measurement even in the narrow cavities (e.g. esophagus) inside the human body (when working with a medical endoscope) (Fig. 1b). However, achieving probe miniaturization and "side-viewing" capability requires dense placement and sharp bending of optical fibers, which would cause excessive light loss, cross-talk or even mechanical fracture of the optical fibers.

To overcome these challenges, we report the design, fabrication and testing of the first micromachined OIDRS probe with "side viewing" capability. The precision and mass production capability of micromachining ensures the required performance, as well as the compact size and low-cost fabrication of the probe. Using this probe, the optical absorption and scattering properties of human esophageal specimen have been successfully characterized ex-vivo, which clearly differentiate cancerous tissues from benign ones.

\section{PRINCIPLE OF OIDRS}

As shown in Fig. 2, when light is incident on the surface of an inhomogeneous medium (e.g. biological tissue), part of the incident light will be directly reflected (forming the specular reflectance) and the remaining will transmit into and interacts with the media. After undergoing scattering and absorption multiples times, a portion of the transmitted light will be "turned back" and escape from the surface the medium, which forms the diffuse reflectance. Diffuse reflectance is strongly dependent on both the absorption and scattering properties of the inhomogeneous medium.

According to the diffusion theory, the one-dimensional spatial distribution of steady-state diffuse reflectance at certain wavelength $\left(R_{d}(x)\right)$ for oblique light incidence can be estimated using a modified two-source approximation with one positive source located below the sample surface and one negative source located above the sample surface (Fig. 3) which is expressed as

$$
R_{d}(x)=\frac{1}{4 \pi}\left[\frac{\Delta z\left(1+\mu_{e f f} \rho_{1}\right) \exp \left(-\mu_{e f f} \rho_{1}\right)}{\rho_{1}^{3}}+\frac{\left(\Delta z+2 z_{b}\right)\left(1+\mu_{\text {eff }} \rho_{2}\right) \exp \left(-\mu_{e f f} \rho_{2}\right)}{\rho_{2}^{3}}\right]
$$

where $\mu_{\text {eff }}$ is the effective attenuation coefficient, $\rho_{1}$ and $\rho_{2}$ are the distances between the two sources and the observation point on the surface of the medium, respectively, $\Delta z$ is the distance between the virtual boundary and the medium depth, and $z_{b}$ is the distance between

Sens Actuators A Phys. Author manuscript; available in PMC 2015 January 07. 
the virtual boundary and the surface of the medium [12]. The absorption coefficient $\left(\mu_{a}\right)$ and reduced scattering coefficient $\left(\mu_{s}^{\prime}\right)$ can be determined as

$$
\begin{gathered}
\mu_{a}=\frac{\mu_{e f f}^{2} \Delta x}{3 \sin \left(\alpha_{t}\right)} \\
\mu_{s}^{\prime}=\frac{\sin \left(\alpha_{t}\right)}{\Delta x}-0.35 \mu_{a}
\end{gathered}
$$

where $\Delta x$ is the shift of the point sources in the $x$ direction for the modified dipole source diffusion theory model, $\mathrm{a}_{t}$ is the angle of light transmission into the medium. By capturing the spatial distribution of diffuse reflectance $\left(R_{d}(x)\right)$ the optical properties $\mu_{a}$ and $\mu_{s}{ }^{\prime}$ can thus be calculated by solving the inverse problem [13]. It should be noted that the diffusiontheory-based model is only valid if the reduced scattering coefficient is much larger than the absorption coefficient [14] (which is the case for biological tissues). Also, the source and detector must be separated such that the light consists of diffuse reflectance when it reaches the detector.

\section{PROBE DESIGN}

To conduct OIDRS measurement of biological tissues, it is necessary to deliver light of particular wavelength at a desirable oblique incidence angle on the tissue surface and also collect the one-dimensional spatial distribution of the diffuse reflectance $\left(R_{d}(x)\right)$. While this can be achieved by placing the biological tissue under a delicate imaging setup, it will not be convenient and feasible to conduct in-vivo OIDRS measurements (especially inside human body) under typical clinical settings. To address this issue, we have investigated the idea of building a standalone fiber optic sensor probe for skin applications [11]. The sensor probe consists of three source fibers ( $200 \mu \mathrm{m}$ in diameter) and two linear arrays of twelve collection fibers $(100 \mu \mathrm{m}$ in diameter and with a pitch of $300 \mu \mathrm{m})$ for capturing the spatial distribution of $R_{d}(x)$ (Fig. 4a). The effective probe testing area is limited to $2 \times 2 \mathrm{~mm}^{2}$ to ensure its capability to measure the diffuse reflectance of even the smallest skin lesions (usually around $3 \times 3 \mathrm{~mm}^{2}$ ). Among the three source fibers, two are used for oblique incidence, delivering light onto the skin surface at an oblique angle (a) of $45^{\circ}$. Since the OIDRS measurement is usually performed in a dark environment to reduce the effect of the background light, the center normal incidence source fiber is used to illuminate area of interest on the skin to ensure the accurate placement of the sensor probe. Although only one oblique incidence fiber and one linear array of collection fibers are necessary for an OIDRS measurement, two oblique incidence fibers and two arrays of collection fibers are used for higher efficiency and effectiveness of data collection. To achieve accurate alignment of the source and collection fibers necessary for robust clinical application, four micromachined positioning devices (two for source fibers and two for collection fibers) were fabricated. Each positioning device consists of a silicon substrate with either photolithography patterned SU-8 guiding structures for the source fibers or bulk etched V-grooves for aligning the collection fibers. After assembling the source and collection fibers, all the positioning devices are stacked and glued together with epoxy. Finally, the assembled probe is mounted into an aluminum probe holder to facilitate the testing (Fig. 4b).

Sens Actuators A Phys. Author manuscript; available in PMC 2015 January 07. 
According to Fig. 1a, the skin probe assumes a "front viewing" configuration, in which the sensor head is in line with the direction of the source/collection fiber bundles. All the collection fibers remain straight and thus can be arranged in a dense manner with suitable spacing for capturing the spatial distribution of the diffuse reflectance. To accommodate the $45^{\circ}$ bending of the two source fiber for oblique incidence, an outer dimension of the probe $\left(15 \times 10 \mathrm{~mm}^{2}\right)$ is used to prevent sharp bending of the source fibers. For in-vivo skin testing, the "front viewing" configuration and the relatively large size of the probe are desirable for hand-held applications. However, for inner-body applications (e.g. esophagus), this configuration and dimension of sensor probe becomes an issue. First, to conduct effective and efficient in-vivo measurement inside the human body, the OIDRS probe has to be mounted onto a medical endoscope for manipulation and targeting. Current medical endoscopes can only accept tool attachments with diameters ranging from 2 to $5 \mathrm{~mm}$. The current OIDRS probe is too bulky for this purpose. Second, while the "front viewing" configuration is ideal for skin applications, it will be difficult to use it to conduct in-vivo measurements in the tight inner-body cavities. A "side viewing" configuration will be preferable, in which the sensor head is oriented in a perpendicular position with respect to the axis of the fiber bundles (see Fig. 1b). However, this inevitably requires a sharp $90^{\circ}$ turn for all the collection fibers within a very tight space, which would cause very high light loss and leakage (with cross-talk between adjacent collection channels) and also possible mechanical fracture of the collection fibers. To address this issue, we have come up with a new 3-chip probe design with lithographically patterned polymer waveguides (Fig. 5). This new probe design consists of three substrates. The first substrate serves as the positioning device to guide the source fiber for a $45^{\circ}$ oblique incidence. To reduce the probe size, only one source fiber is used for oblique incidence. Since the endoscope already has built-in light source for illumination, the normal incidence fiber which was used in the skin probe will not be necessary. To avoid the detrimental effects of direct sharp bending of the collection fiber array, a second substrate with microfabricated polymer waveguides is used for collecting the diffuse reflectance. The four side walls of curved polymer waveguides are coated with highly reflective layers to prevent possible light leakage and cross-talk of the waveguides. To interface the waveguides to OIDRS image capture and processing setup, a third substrate with bulk-etched $\mathrm{V}$-groves is used to align the straight interconnection fiber bundles with exactly same pitch as that of the polymer waveguides.

\section{FABRICATION AND ASSEMBLY}

The OIDRS probe fabrication and assembly process consists of two steps: (1) fabrication of the three positioning substrates and (2) assembly of the source/collection fibers and device substrates. We chose SU-8 resist (MicroChem, MA) as structural materials for both the guiding structures of source fiber and also the curved waveguides for collecting the diffuse reflectance. First, SU-8 can be directly lithographically patterned to form thick and highaspect-ratio microstructures $(100 \sim 1000 \mu \mathrm{m})$. Second, although it is not one of the most optically transparent polymer materials, SU-8 can still provide acceptable optical transmittance over the visible light spectrum for conducting OIDRS (Fig. 6). The use of SU-8 microstructures eliminates the need of expensive microfabrication equipment and complex process steps (e.g. deposition and etching), which results in a straightforward and 
low-cost fabrication process. This is important for future clinical application of OIDRS since the probe will be the only consumable part of the entire OIDRS system and it will be highly desirable to make it low-cost and thus disposable after a certain amount of use.

To align the source fiber ( $200 \mu \mathrm{m}$ in diameter) to the $45^{\circ}$ oblique incidence angle, the first micro positioning substrate chip with $100-\mu$ m-thick SU-8 guide structures was fabricated. SU-8 100 resist was spun on a pre-cleaned silicon wafer (500 $\mu \mathrm{m}$ thick) at calibrated spinning rate to reach a final thickness of about $100 \mu \mathrm{m}$. After the spin-coating of SU-8, a process recipe recommended by the manufacturer was followed to make the guide structure with a 200- $\mu$ mwide curved trench (Fig. 7a) [15]. During the SU-8 processing, a slow temperature ramping was maintained to minimize the internal stress build-up and also the resulting crack formation within the SU-8 film, which would significantly reduce the mechanical strength and stability of the guiding structures. Next, the above SU-8 process was repeated on another silicon wafer to make the $90^{\circ}$ curved collection waveguides (10 channels) with a cross-section area of $100 \times 100 \mu \mathrm{m}^{2}$ each (Fig. 7b). To minimize light leakage and cross-talk between adjacent collection channels, the top, bottom and two side surfaces of the rectangular SU-8 waveguides were coated with thermally-evaporated aluminum ( $300 \mathrm{~nm}$ thick) in three consecutive steps. Before the SU-8 waveguide structures were formed, the bottom aluminum layer was deposited on the substrate. After the SU-8 step, two more aluminum depositions were conducted with the substrate placed at an oblique $\pm 20^{\circ}$ with respect to the evaporation source to ensure a good coverage of the two side walls and the top surface. Finally, to accurately align the interconnection optical fibers $(100 \mu \mathrm{m}$ in diameter), the third micro positioning substrate was fabricated, which consists of a silicon substrate with a linear array of V-grooves created with silicon bulk etching (Fig. 7c). When an optical fiber (with cylindrical cross-section) is placed in a V-groove, the center axis of the optical fiber can "automatically" align with the symmetric plane of the V-groove. Thus, the accurate positioning of the intercollection fibers can be readily achieved to ensure reliable and uniform performance of the sensor probe. To fabricate the positioning chip, silicon nitride was deposited on a $\{100\}$ silicon wafer. Photolithography and reactive ion etching was conducted to pattern the silicon nitride layer, which serves as a hard mask for silicon bulk etching. Silicon bulking etching was performed in potassium hydroxide solution to form the $\mathrm{V}$-grooves $(230 \mu \mathrm{m}$ wide and $300 \mu \mathrm{m}$ pitch).

After the fabrication, the probe assembly process was conducted. The source incidence fiber was carefully placed into the SU-8 alignment structure on the micro positioning substrate. A slow drying black epoxy was used to fix it in place (Fig. 8a). The same procedure was repeated for assembling the interconnection fibers. To improve the efficiency of light incidence and collection of the optical fibers and SU-8 waveguides, the edge of the silicon chips with either input/output end of the fibers or waveguides were polished first with $2 \mu \mathrm{m}$ and then $0.3 \mu \mathrm{m}$ sand paper. After the polishing, the SU-8 collection waveguides were aligned with the interconnection fibers. The position of the SU-8 waveguide chip was adjusted to couple the maximum amount of light coming out from the distal end of SU-8 waveguides. Once this was achieved, black epoxy was applied to bond the two chips together. The assembly of this probe is completed by stacking and gluing the source fiber substrate with the SU-8 waveguide substrate. The tip of the incidence fiber was visually aligned to the center location of SU- 8 waveguide array. The assembled probe was then 
placed in an aluminum holder to facilitate the probe testing (Fig. 8b). The overall dimension of the sensor probe is $5 \times 5 \times 12 \mathrm{~mm}^{3}$, which should be small enough and suitable for ex-vivo esophagus measurements (Fig. 8c). .

\section{OIDRS SYSTEM}

To conduct OIDRS measurement, we have built a complete experimental setup to interface with the developed sensor probe to achieve automated optical incidence control, data collection and analysis (Fig. 9). It consists of a white light source (halogen lamp) for multiple-wavelength measurement, multiplexer, imaging spectrograph, CCD camera, and personal computer. The 16- bit CCD camera (Andor Technology 412DV) has a 512×512pixel CCD chip with dimensions of $12.6 \times 12.6 \mathrm{~mm}^{2}$. The imaging spectrograph (Oriel MS257) has a 150-lines/mm grating. With this combination of chip size and grating we are able to image a wavelength in the range of $310 \mathrm{~nm}$ onto the CCD matrix. Before an OIDRS measurement is conducted, the source fiber of the sensor probe is connected to the output of the light source via an SMA connector. The proximal end of each of the intercollection fibers is also fitted with SMA 905 connectors and then connected to the input of the spectrograph through a custom-made multitrack bundle. The optical multiplexer allows the light delivery, to the area of interest, through only one source fiber at a time. After the sensor probe gets into contact with the sample surface, white light is delivered through the source fiber and the diffuse reflectance is then captured by the collection waveguides and coupled through the interconnection optical fibers to the imaging spectrograph that generates an optical spectrum for each collection channel. The CCD camera collects the spectralimages from the wavelength range of 455 to $765 \mathrm{~nm}$. The spectral images represent the steady-state diffuse reflectance spectra from each collection channel, which are stored in the computer for further analysis. This entire OIDRS system is capable of capturing one frame of spectral image in a fraction of a second.

Before the actual OIDRS measurement was conducted, the experimental setup was calibrated and validated using a liquid reference solution (phantom) consisting of polystyrene microspheres as scattering elements and trypan blue as absorber [11]. The absorption coefficient spectra of trypan blue were measured by collimated transmission before mixing it with the polystyrene micro-spheres. The reduced scattering coefficient of the microspheres was calculated using Mie theory [16]. The "expected values" of the absorption and reduced scattering coefficients of the liquid reference solution can be varied by controlling the concentration of absorbing and scattering chemicals.

To conduct the calibration, the sensor probe was placed on the surface of the reference solution. The probe was rotated to four different angles with respect to an arbitrary reference point, and the diffuse reflectance was recorded each time. The absorption and reduced scattering spectra were extracted for each diffuse reflectance measurement and averaged to obtain the "estimated values". The system was calibrated by measuring several optical reference phantoms. The reading from each collection fiber channel is compensated by a factor that matches the measured diffuse reflectance to their expected values. After the calibration, the probe was tested with different reference solution. The Fig. 10 shows the average measured optical properties and the standard deviation for those measurements. 


\section{TESTING AND RESULTS}

After the calibration, the entire OIDRS system was used to conduct ex-vivo measurement of human esophagus specimens with cancerous tissues (adenocarcinoma) for the first time (one specimen shown as an example) (Fig. 11). Only the center eight collection channels are used in order to reduce the effective probe contact area to $1.6 \times 1 \mathrm{~mm}^{2}$ for more accurate measurements. The measurement results clearly show different diffuse reflectance spectra for the normal and cancerous tissues (Fig. 12). The general lower diffuse reflectance of the cancerous tissue is caused by its darker color (causing more light absorption). Cancerous tissue is believed to have relatively larger nuclei causing stronger optical scattering serving as a good indicator for distinguishing it from benign cells [17].

\section{CONCLUSIONS AND DISCUSSION}

A new miniaturized OIDRS sensor probe has been successfully developed and used for exvivo testing of esophageal cancer for the first time. The further miniaturization of the probe will allow its operation with medical endoscopes, to enable in-vivo non-invasive detection of esophageal cancer and possibly many other epithelial types of cancers inside human body.

In OIDRS, the use of multiple collection channels allows capturing the 1D spatial distribution of the diffuse reflectance. This approach partly overcomes one of the greatest perceived weaknesses of single-channel elastic scattering spectroscopy, and thus enables fundamental optical properties of absorption and scattering to be individually resolved. Since the optical absorption and scattering of human tissues are closely related to some of the key diagnostic physiological parameters of malignancy, OIDRS is capable of classifying cancerous tissues from benign ones with high sensitivity and accuracy, which would excel other competing optical spectroscopic methods, such as fluorescence spectroscopy. This result is backed by our clinical study on the classification of the skin lesion. Using the developed OIDRS probes, we plan to conduct an extensive study on the classification of different types of esophageal lesions to further verify its sensitivity and reliability. Once available, the results of this study will be presented in future publications.

\section{ACKNOWLEDGMENTS}

This project is partially supported by National Institutes of Health under contract R01 CA106728.

\section{REFERENCES}

1. American Cancer Society website. What Are the Key Statistics About Cancer of the Esophagus?. 2008 http://www.cancer.org.

2. Wang HW, Putt ME, Emanuele MJ, Shin DB, Glatstein E, Yodh AG, Busch TM. TreatmentInduced Changes in Tumor Oxygenation Predict Photodynamic Therapy Outcome. Cancer Res. 2004; 64:7553-7561. [PubMed: 15492282]

3. Bargo PR, Prahl SA, Goodell TT, Sleven RA, Koval G, Blair G, Jacques SL. In vivo determination of optical properties of normal and tumor tissue with white light reflectance and an empirical light transport model during endoscopy. J. Biomed. Opt. 2005; 10:034018. [PubMed: 16229662]

4. His RA, Rosenthal DI, Glatstein E. Photodynamic therapy in the treatment of cancer: current state of the art. Drugs. 1999; 57:725-734. [PubMed: 10353297] 
5. Troy TL, Page DL, Sevick-Muraca EM. Optical properties of normal and diseased breast tissues: prognosis for optical mammography. J. Biomed. Opt. 1996; 1:342-355. [PubMed: 23014735]

6. Mayinger B, Horner P, Jordan M, Gerlach, Dipl.Phys. C, Horbach T, Hohenberger W, Hahn EG. Endoscopic fluorescence spectroscopy in the upper GI tract for the detection of GI cancer: initial experience. Am. J. Gastroenterol. 2001; 96:2616-2621. [PubMed: 11569684]

7. Brand S, Poneros JM, Bouma BE, Tearney GJ, Compton CC, Nishioka NS. Optical Coherence Tomography in the Gastrointestinal Tract. Endoscopy. 2000; 32:796-803. [PubMed: 11068841]

8. Messmann H, Knuchel R, Baumler W, Holstege A, Scholmerich J. Endoscopic fluorescence detection of dysplasia in patients with Barrett's esophagus, ulcerative colitis, or adenomatous polyps after 5-aminolevulinic acid-induced protoporphyrin IX sensitization. Gastrointest Endosc. 1999; 49:97-101. [PubMed: 9869731]

9. Wang L-H, Jacques SL. Use of a laser beam with an oblique angle of incidence to measure the reduced scattering coefficient of a turbid medium. Appl. Opt. 1995; 34:2362-2366. [PubMed: 21037790]

10. Garcia-Uribe A, Kehtarnavaz N, Marquez G, Prieto V, Duvic M, Wang LV. Skin cancer detection by spectroscopic oblique-incidence reflectometry: classification and physiological origins. Appl. Opt. 2004; 43:2643-2650. [PubMed: 15130003]

11. Garcia-Uribe, A.; Hong, J-M.; Zou, J.; Wang, LV. Micromachined Oblique Incidence Reflectometry (OIR) Probe for Skin Cancer Detection; Proceedings of IEEE Transducers'07; Lyon, Franc. 2007. p. 1099-1102.

12. Marquez G, Wang L-H. White light oblique incidence reflectometer for measuring absorption and reduced scattering spectra of tissue-like turbid media. Opt. Express. 1997; 1:454-460. [PubMed: 19377569]

13. Lin S-P, Wang L, Jacques SL, Tittel FK. Measurement of tissue optical properties by the use of oblique-incidence optical fiber reflectometry. Appl. Opt. 1997; 36:136-143. [PubMed: 18250654]

14. van de Hulst, HC. Light Scattering by Small Particles. New York: Dover Publications, Inc.; 1981.

15. Microchem website, NanoTM SU-8. http://www.microchem.com.

16. Farrell TJ, Patterson MS. A diffusion theory model of spatially resolved, steady-state diffuse reflectance for the noninvasive determination of tissue optical properties in vivo. Med. Phys. 1992; 19:879-888. [PubMed: 1518476]

17. Backman V, Wallace MB, Perelman LT, Arendt JT, Gurjar R, Muller MG, Zhang Q, Zonios G, Kline E, McGillican T, Shapshay S, Valdez T, Badizadegan K, Crawford JM, Fitzmaurice M, Kabani S, Levin HS, Seiler M, Dasari RR, Itzkan I, Van Dam J, Feld MS. Detection of preinvasive cancer cells. Nature. 2000; 406:35-36. [PubMed: 10894529] 


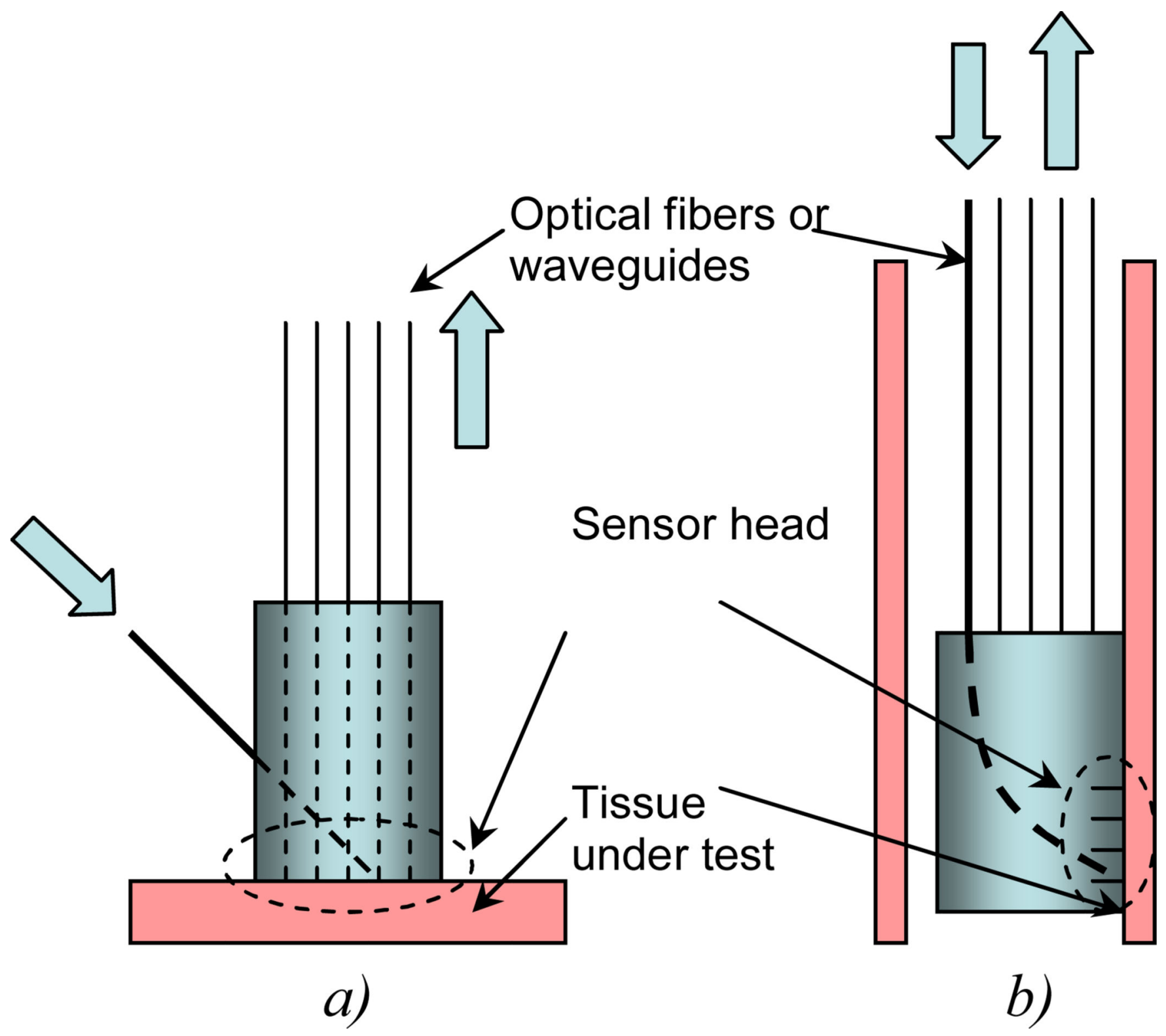

Fig. 1.

Schematic of OIDRS probes: (a) front viewing and (b) side viewing. 


\section{Incident Light}

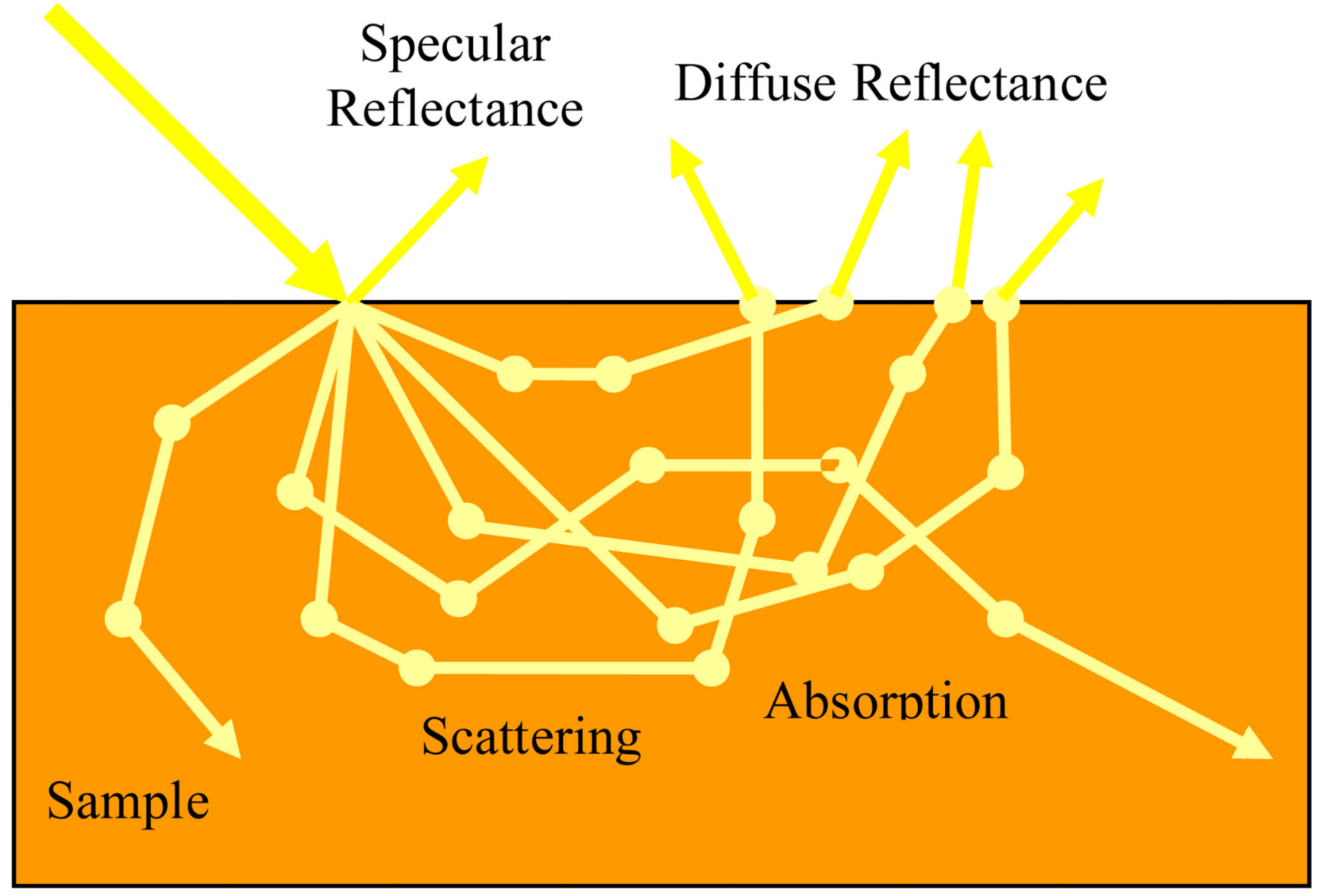

Fig. 2.

Illustration of the absorption and scattering of oblique light incidence in an inhomogeneous medium. 


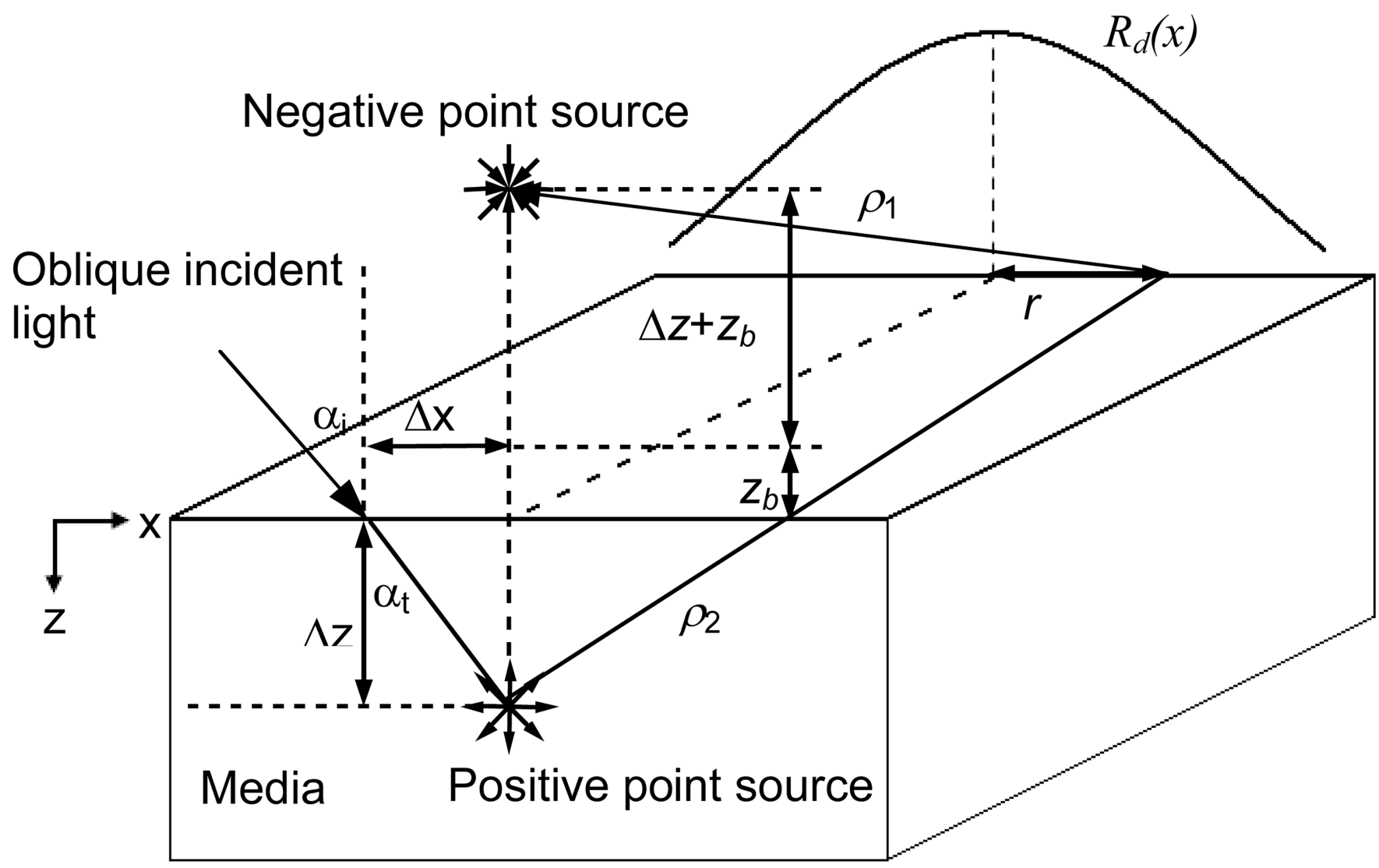

Fig. 3.

Schematic of the modified two-source approximation of oblique incidence based on the diffusion theory. 


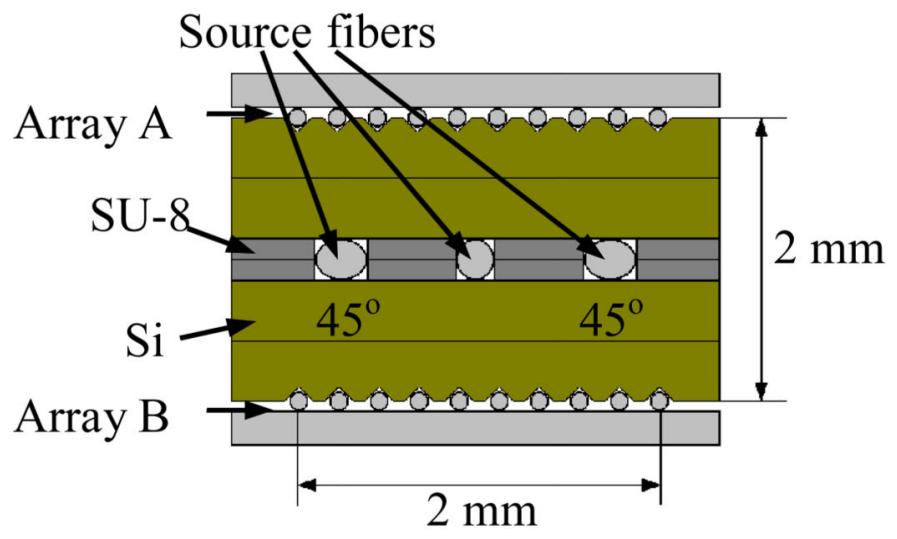

(a)

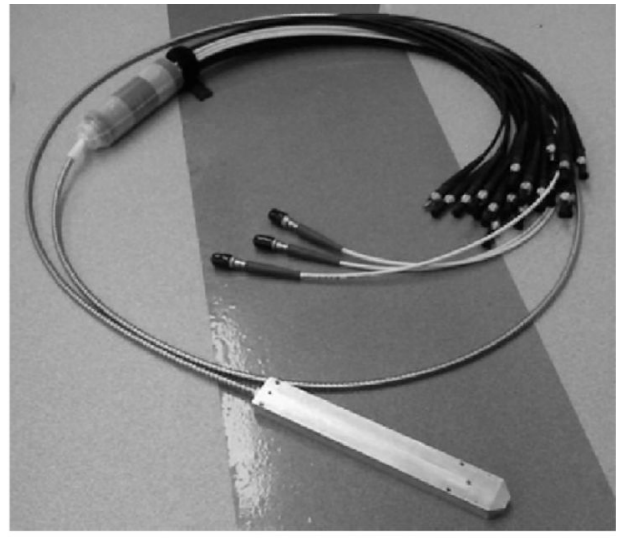

(b)

Fig. 4.

(a) Schematic of the front view of the OIDRS probe for in-vivo skin testing; (b) The complete probe assembly. 


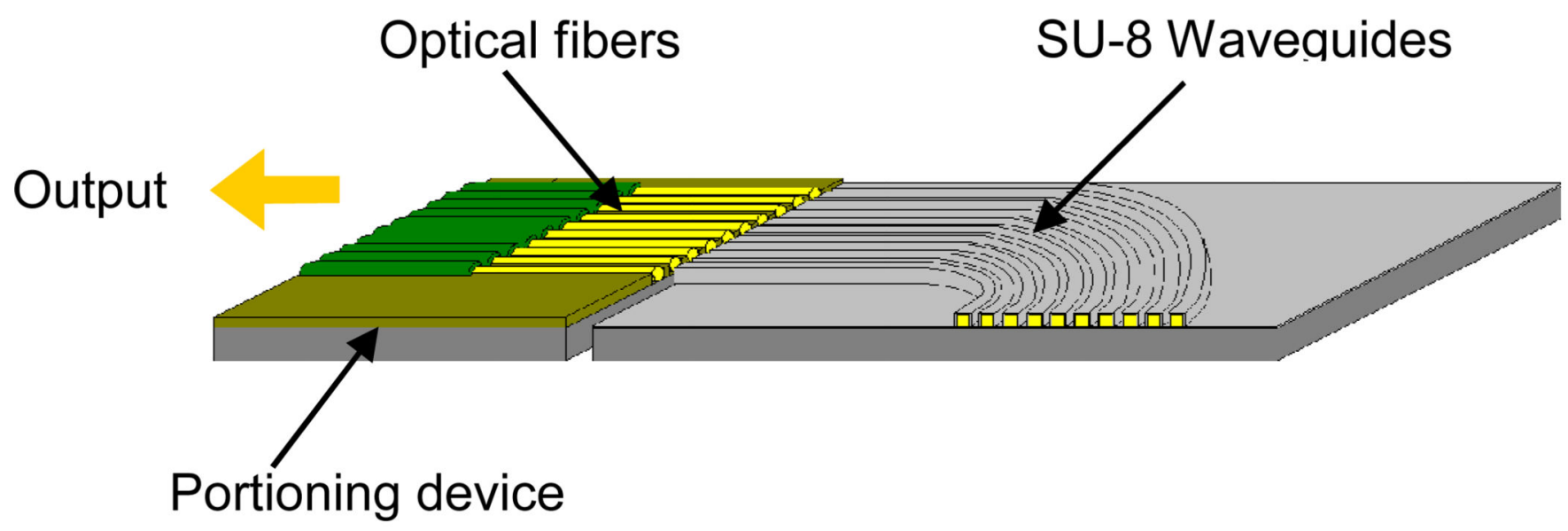

Input

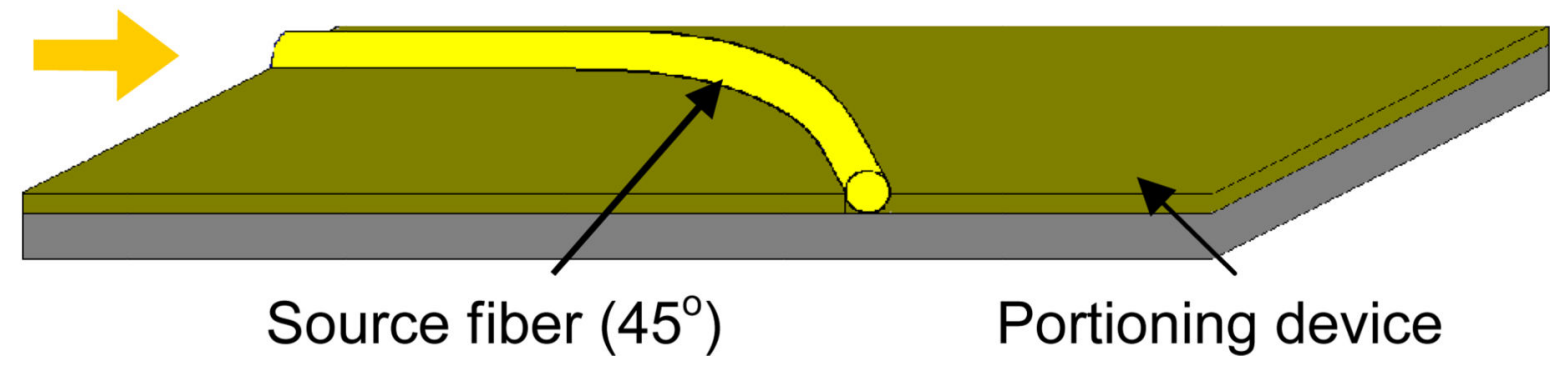

Fig. 5.

Schematic design of the "side-viewing" OIDRS probe. 


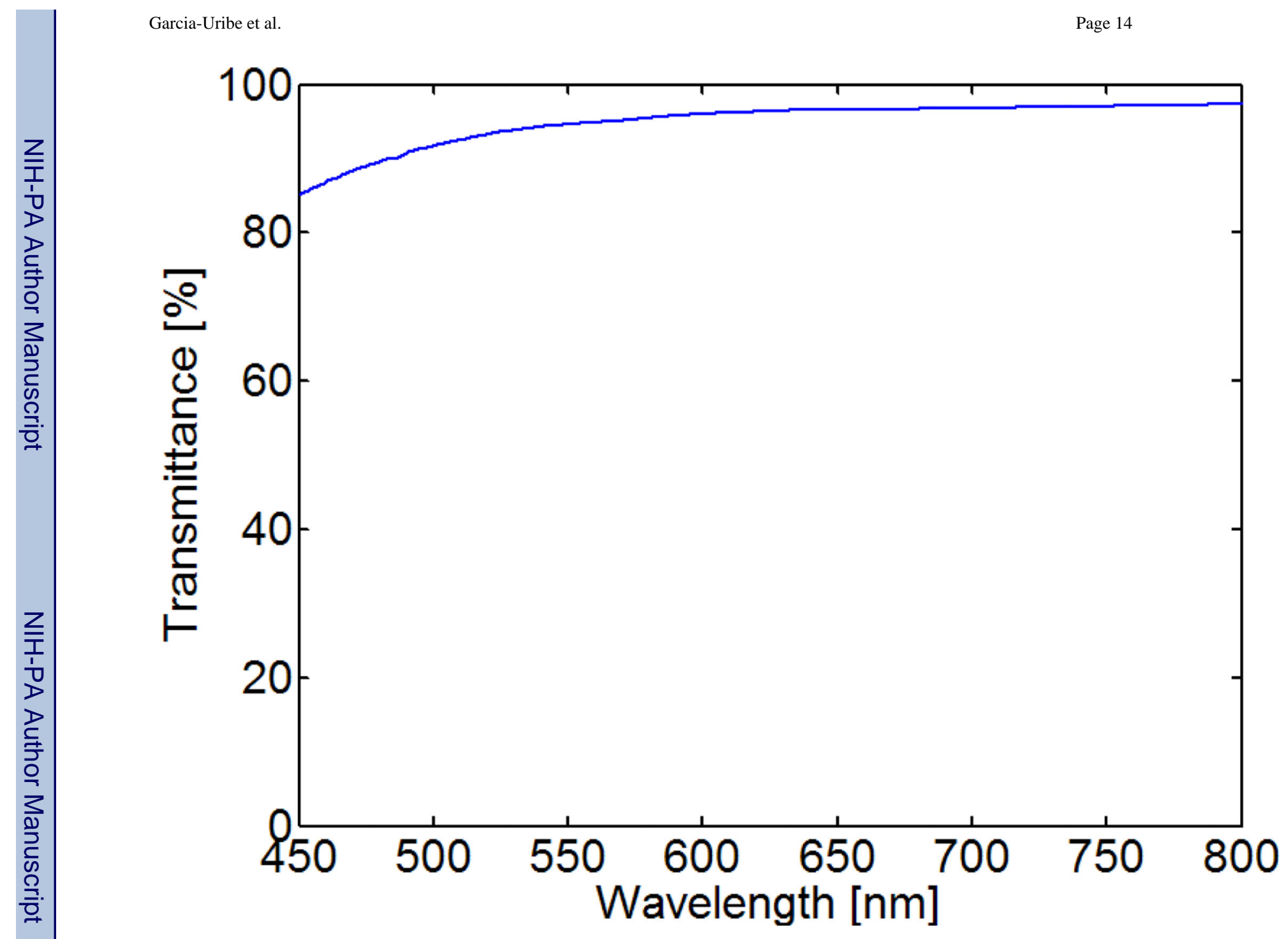

Fig. 6.

Optical transmittance of a cured SU-8 film (100 $\mu \mathrm{m}$ thick) from $450 \mathrm{~nm}$ to $800 \mathrm{~nm}$, which is characterized using a white light source and a single channel spectrometer 


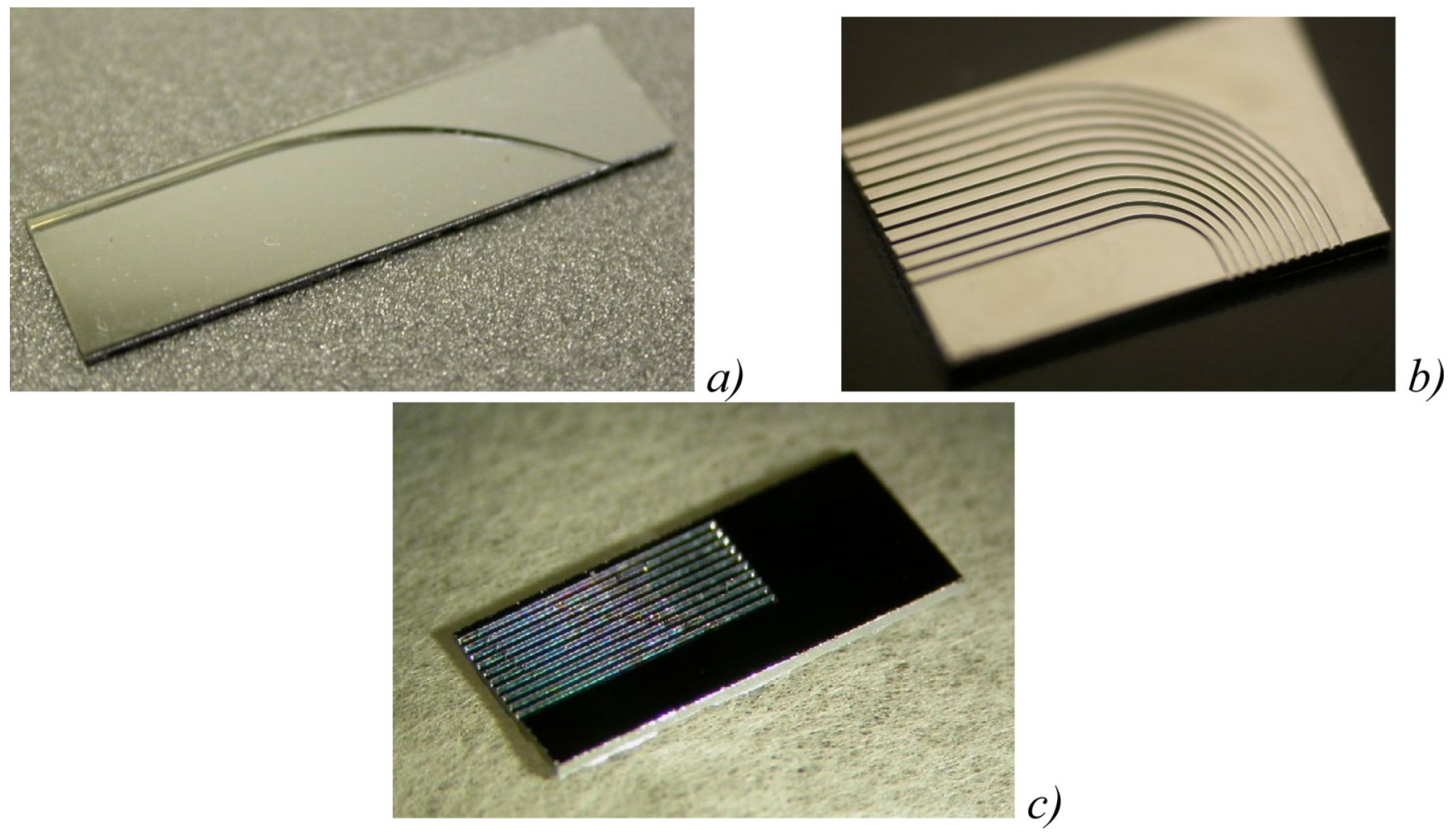

Fig. 7.

Micromachined silicon positioning substrates for the OIDRS probe: (a) Source fiber guide; b) Collection waveguide substrate; and c) Interconnection fiber guide. 


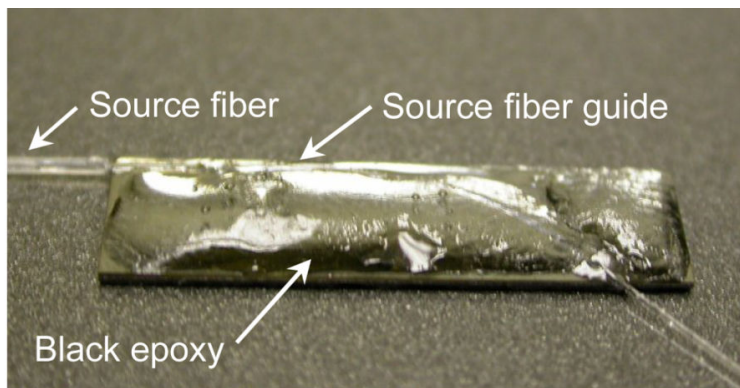

a)
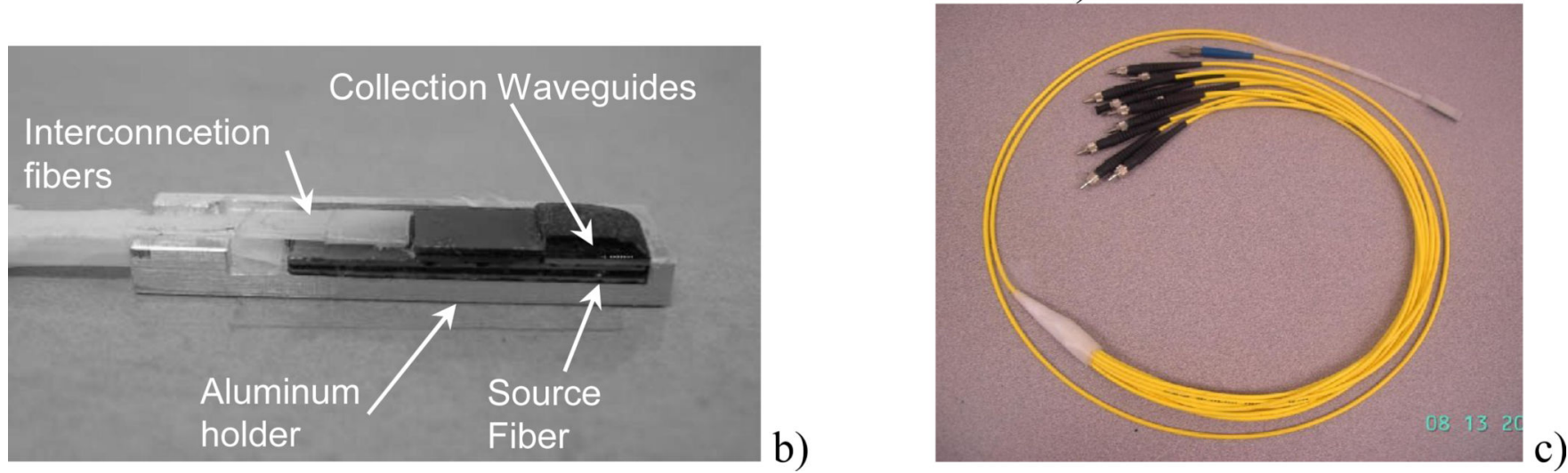

Fig. 8.

OIDRS probe assembly: a) Source fiber; b) Assembled sensor probe; and c) Complete sensor probe with optical adapter. 


\section{Personal Computer \\ Light}

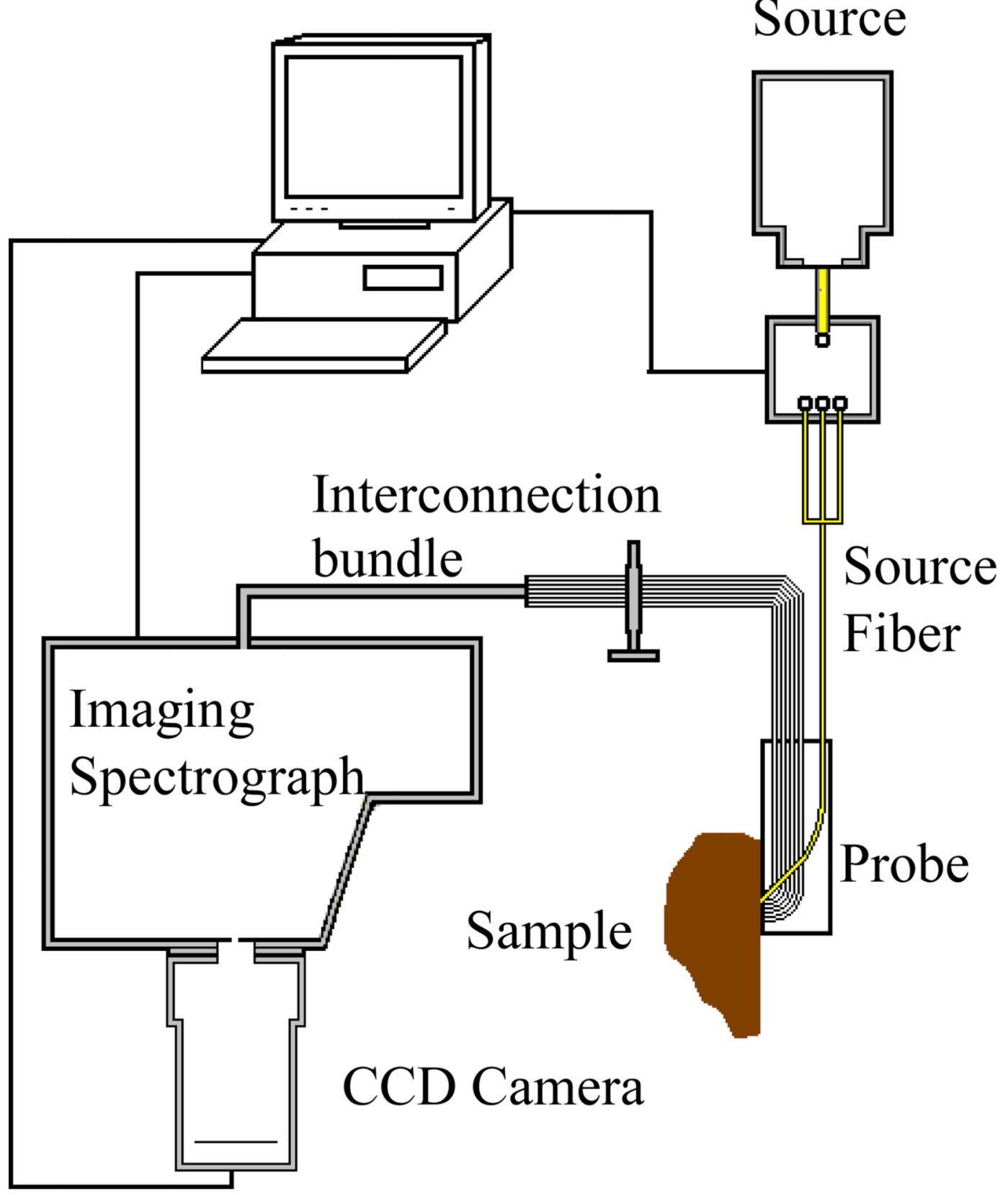

Fig. 9.

Experimental setup for OIDRS probe testing.

Source 


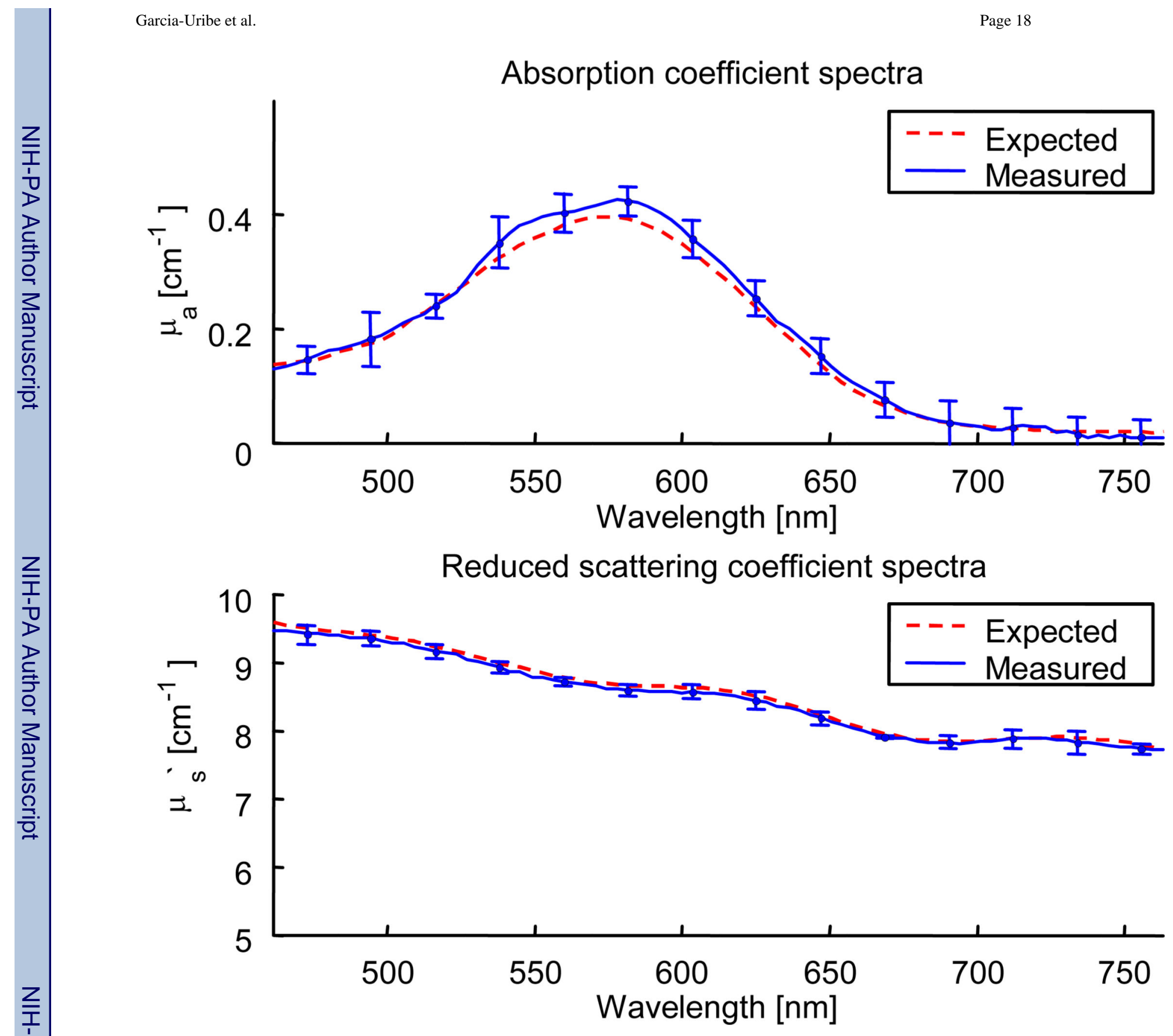

Fig. 10.

Expected and measured absorption and reduced scattering spectra of a liquid reference solution. 


\section{Normal}

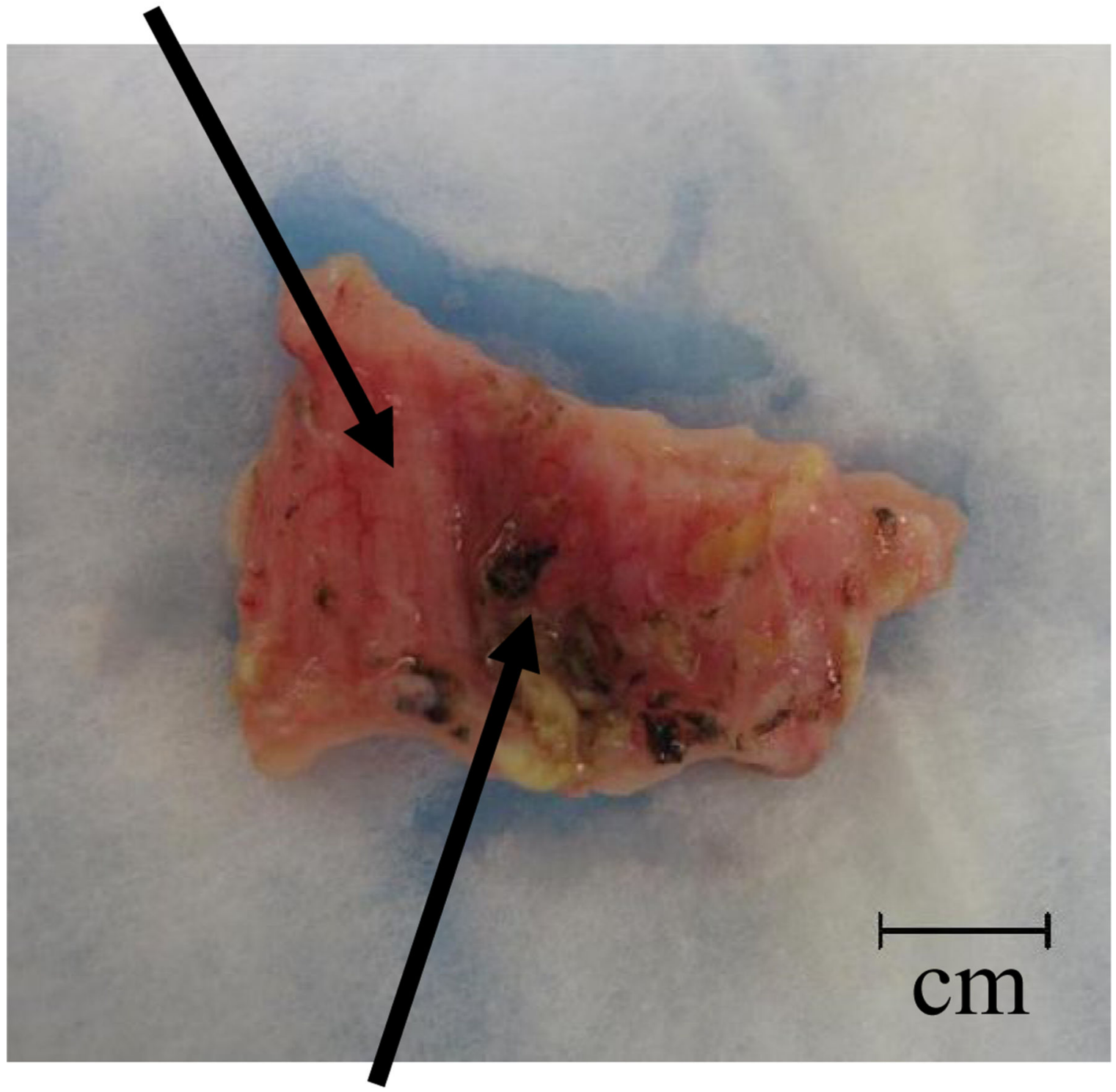

\section{Cancerous}

Fig. 11.

A human esophagus specimen with cancerous tissue (adenocarcinoma). 


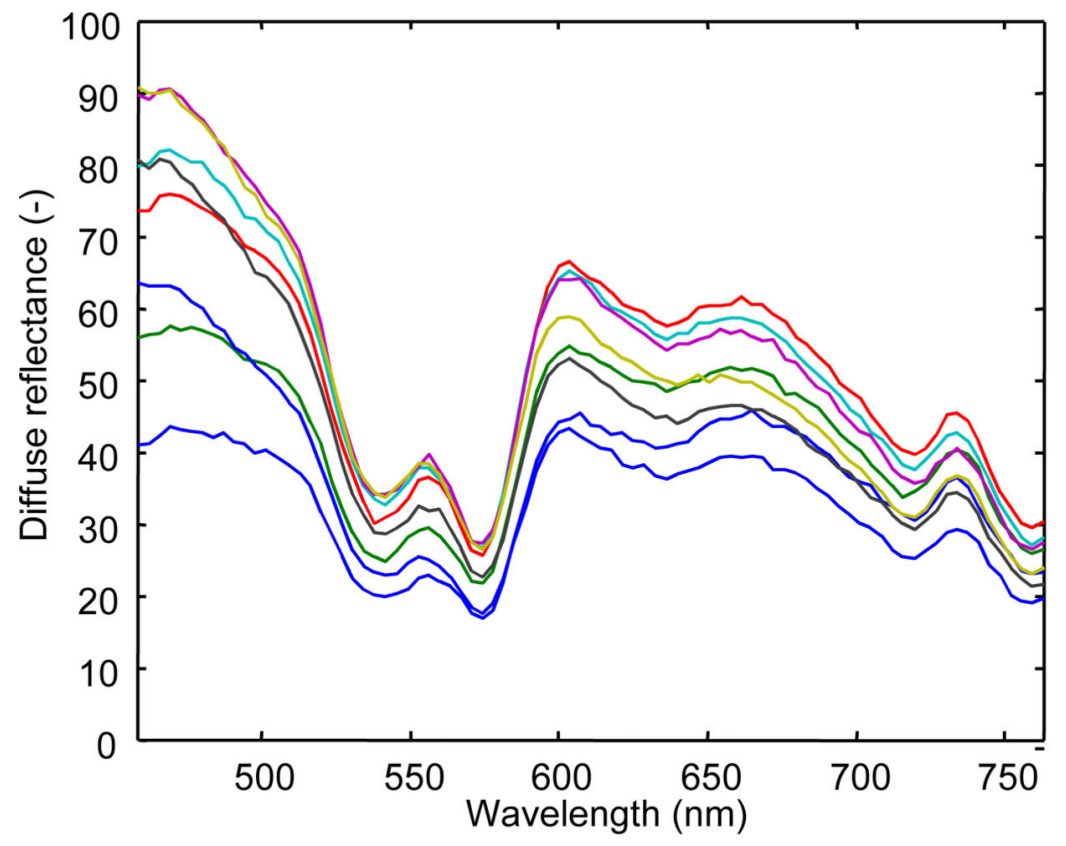

(a)

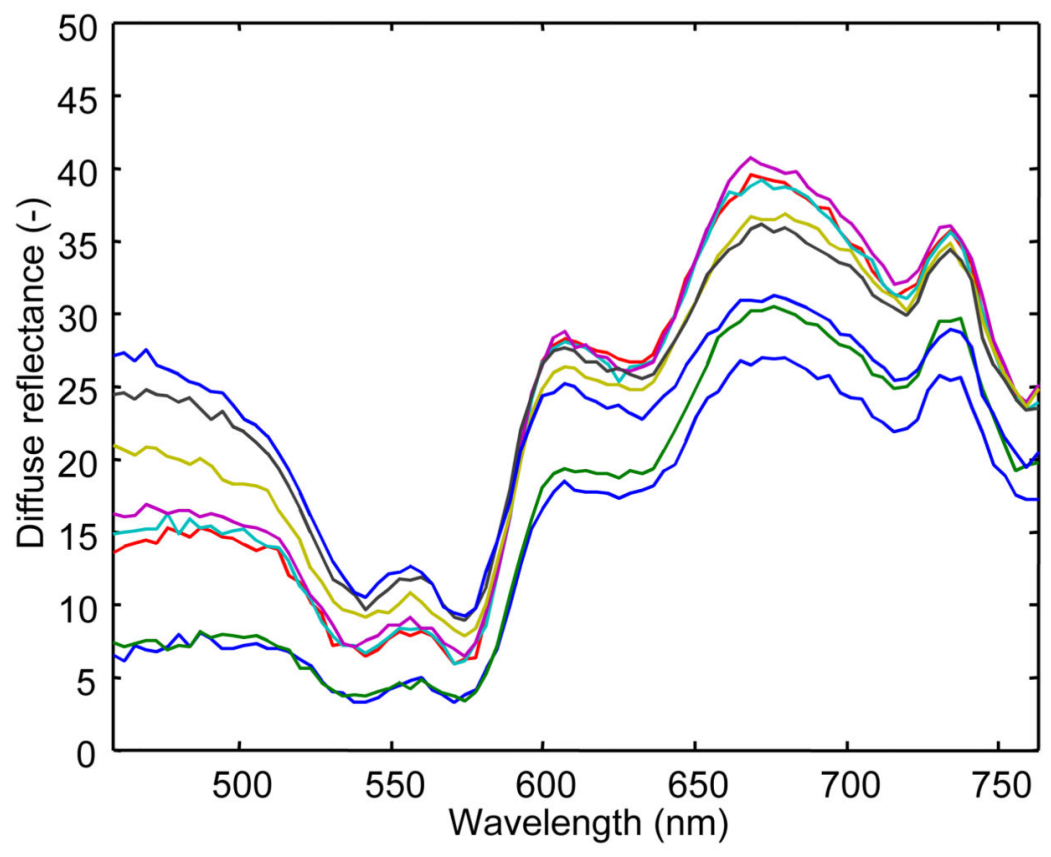

(b)

Fig. 12.

Ex-vivo diffuse-reflectance spectra of the human esophagus specimen shown in Fig. 11: (a) Normal tissue; and (b) Cancerous tissue. 